\title{
Genetic Algorithms for Positron Lifetime Data
}

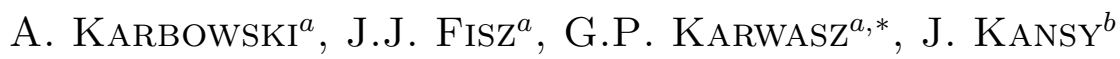 \\ AND R.S. BRUSA ${ }^{c}$ \\ ${ }^{a}$ Institute of Physics, Nicolaus Copernicus University \\ Grudziądzka 5, 87-100 Toruń, Poland \\ ${ }^{b}$ Institute of Physics, Silesian University \\ Bankowa 12, 40- 007 Katowice, Poland \\ ${ }^{c}$ Dipartimento di Fisica, Universitá di Trento \\ Via Sommarive 14, I-38050 Povo, Trento, Italy
}

\begin{abstract}
Recently, genetic algorithms have been applied for ultrafast optical spectrometry in systems with several convoluted lifetimes. We apply these algorithms and compare the results with POSFIT (by Kirkegaard and Eldrup) and LT programme (by Kansy). The analysis was applied to three types of samples: molybdenum monocrystals, Czochralski-grown silicon with oxygen precipitates, Si with under-surface cavities obtained by $\mathrm{He}+\mathrm{H}$ ion co- implantation. In all three tests, the genetic algorithm performs very well, in particular for short lifetimes. Further developments to model the resolution function in genetic algorithms are needed.
\end{abstract}

PACS numbers: 71.60.+z, 78.70.Bj, 71.55.Cn

\section{Introduction}

Positron annihilation techniques are a versatile tool for identifying the type of defects in semiconductors, see e.g. [1]. Values of lifetimes for pure and defected elements can be calculated with high precision, see e.g. [2] for Si. On the other hand, numerous measurements [3-5] still differ in precise determination of these lifetimes. One reason for the discrepancies are differences in experimental setups, another can be details of the numerical procedures used for analysis of the lifetime spectra.

Recently [6], genetic algorithms have been applied to the analysis of multicomponent fluorescence spectra in complex samples. In this work we develop

*corresponding author; e-mail: karwasz@fizyka.umk.pl 
procedures for applying these algorithms for positron lifetime spectroscopy. Genetic algorithms have been recently applied to numerous scientific subjects, ranging from catalyst design [7], protein structures [8] to search for extra-solar planets [9]. As far as we know, no attempt has been to apply these algorithms for positron lifetime spectra. Therefore, we test this method for three cross-check cases:

1) lifetime spectra in pure, defect free monocrystal molybdenum samples measured with good (169 ps) resolution [10];

2) lifetime spectra in high-purity Czochralski-grown silicon ( $\mathrm{Cz}-\mathrm{Si})$ in which oxygen precipitates were induced by thermal treating at high hydrostatic pressures; our previous analysis of these spectra [11] with POSFIT method [12] indicated presence of nano-voids with lifetimes in a broad range (0.5$1.1 \mathrm{~ns})$

3) silicon samples in which nanocavities were formed by $\mathrm{He}^{+}$and successive $\mathrm{H}^{+}$ion implantation [13]; these spectra were obtained with the use of depthprofiling (positron beam) technique, with about 250 ps resolution.

Present method of analysis of lifetime spectra combines the genetic algorithm with multiple linear regression (GA-MLR) method. For all three types of samples we compare GA-MLR results with two other methods traditionally used in positron lifetime analysis, POSFIT [12] by Kirkegaard and Eldrup and LT by one of present authors [14].

\section{Numerical method}

The POSFIT package [12] the most widely used in lifetime measurements is based on the convolution method, i.e. constructs the trial function from expected guessed lifetimes and the expected instrument function. The LT allows analysis of con-tinuous distributions of lifetimes and is based on the de-convolution approach $[14,15]$.

The GA-MLR optimizer has been designed [6] for the nonlinear least-squares problems, in which the model function are linear combinations of nonlinear functions. GA optimizes the nonlinear parameters and the linear parameters are calculated from multiple linear regression. In general, optimizing by GA consists in narrowing the range of the variability of the fitted parameters in a way in which biological populations are selected, i.e. by employing two genetic operators crossover and mutation. GA-MLR is an intuitive optimization approach and it exploits all advantages of the genetic algorithm technique. The MLR method is embedded in the GA optimizer and linear and nonlinear model parameters are optimized in parallel.

In the GA-MLR optimization approach, the GA optimizer provides a trial population of vectors $\boldsymbol{q}$ (i.e., a trial population of the complete sets of the nonlinear parameters in $F_{k}(\boldsymbol{q}, x)(k=1, \ldots, M)$. Each trial vector $\boldsymbol{q}$ returns the corresponding $\chi^{2}(\boldsymbol{q}, \boldsymbol{s})$ function of vector $\boldsymbol{s}$ of the combination parameters $a_{k}$ standing at the 
corresponding function $F_{k}(\boldsymbol{q}, x)$, namely

$$
\chi^{2}(q, s)=\frac{1}{v_{\mathrm{f}}} \sum_{i=1}^{N} \frac{1}{\sigma_{i}^{2}}\left[y_{i}-\sum_{k=1}^{M} a_{k} F_{k}(q, x)\right]^{2},
$$

where $v_{\mathrm{f}}$ is the number of freedom.

All $a_{k}$ can be determined from the MLR method, i.e. from the minimumvalue condition of $\chi^{2}(\boldsymbol{q}, \boldsymbol{s})$ with respect to $s$, that is

$$
\frac{\partial \chi^{2}(q, s)}{\partial s}=0 .
$$

This condition leads to the following set of equations:

$$
\sum_{k=1}^{M}\left[\sum_{i=1}^{N} \frac{F_{j}\left(q, x_{i}\right) F_{k}\left(q, x_{i}\right)}{\sigma_{i}^{2}}\right] a_{k}-\sum_{i=1}^{N} \frac{y_{i} F_{j}\left(q, x_{i}\right)}{\sigma_{i}^{2}}=0,
$$

where $j=1, \ldots, M$, which is equivalent to the matrix equation

$$
A s=c,
$$

where $s$ is a column vector of the amplitudes $a_{k}$ and,

$$
A_{j k}=\sum_{i=1}^{N} \frac{F_{k}\left(q, x_{i}\right) F_{j}\left(q, x_{i}\right)}{\sigma_{i}^{2}}, \quad c_{j}=\sum_{i=1}^{N} \frac{y_{i} F_{j}\left(q, x_{i}\right)}{\sigma_{i}^{2}} .
$$

A formal solution to Eq. (4) reads

$$
s=A^{-1} c .
$$

Substituting the obtained values of the amplitudes $a_{k}$ into Eq. (1) and repeating this procedure over all trial vectors $\boldsymbol{q}$, the trial set of the values of $\chi^{2}(\boldsymbol{q}, \boldsymbol{s})$ is obtained. As temporarily the "best" vectors, $\boldsymbol{q}$ and $\boldsymbol{s}$ in the population are considered to be those that correspond to the lowest $\chi^{2}$ value in the set of all $\chi^{2}(\boldsymbol{q}, \boldsymbol{s})$ obtained. The GA optimizer keeps for further reproduction those vectors $\boldsymbol{q}$ in the population that, together with the corresponding vectors $\boldsymbol{s}$ (estimated from the MLR method), return better $\chi^{2}(\boldsymbol{q}, \boldsymbol{s})$ values. The less "fit" vectors $\boldsymbol{q}$ are replaced by new randomly generated individuals. This procedure is repeated iteratively $\chi^{2}$ until reaches a predefined tolerance or the number of iterations (generations) reaches its predefined maximum. Finally, one obtains the coordinates of a probable minimum value $\chi^{2}(\boldsymbol{q}, \boldsymbol{s})$ and its coordinates $\boldsymbol{q}_{\mathrm{m}}$ and $\boldsymbol{s}_{\mathrm{m}}$ in the space of the allowed values of $\boldsymbol{q}$ and $\boldsymbol{s}$.

This method of optimization is similar both in LT [14] and GA-MLR, with the difference that LT uses the double methodology, i.e. the non-linear parameters by the MINSQ procedure [14] and the linear ones outside MINSQ from Eqs. (5). In LT the values of the initial parameters (vector $\boldsymbol{q}$ ) are postulated and for these values optimum parameters of $a_{i}$ (vector $s$ ) are chosen from Eq. (6). For $s$ obtained in this way MINSQ calculates new values of $\boldsymbol{q}$. Then, from Eq. (6) new optimum values of $s$ are calculated until a satisfactory value of $\chi^{2}$ is obtained. In the second stage all parameters (i.e. both linear and non-linear) are treated as non-linear, and fitted by MINSQ. 
The implementation of GA-MLR developed for fluorescence spectroscopy [6] to positron lifetime spectra finds an essential difference with POSFIT and LT as far as the resolution function is considered. The GA-MLR program, working with much slower signals assumes the spectrometer resolution function infinitely narrow. We mimic the resolution function in the GA-MLR program using a "laser pulse" option, in the first approximation as a single Gaussian, with an adjustable position, width, and amplitude. The convolution with the experimental spectrum is done outside the GA-MLR program. Obviously, this does not correspond strictly to the parameters of the resolution function as defined in POSFIT or LT programs but, as proved by present results, it works rather well, in particular with short lifetimes.

\section{Experimental}

Spectra of Mo and $\mathrm{Cz}-\mathrm{Si}$ were obtained at Trento laboratory with a fast-fast coincidence spectrometer with about 169 ps resolution [10]. The total counting rate was about $1.5-2.0 \times 10^{6}$ for Mo and $1.1 \times 10^{6}$ for Si samples; for the latter case the statistics was improved by performing several independent runs with the same sample [11].

Data on $\mathrm{He}+\mathrm{H}$ implanted Si coming from the spectrometer at Universitt Bundeswehr in Mnchen was used with about 230 ps resolution [13]. This spectrometer is based on the beam setup and uses the bunching pulse as the start signal. Positrons probe different depths of the material, according to their implantation energy. The total counting rate for these measurements was about $1.2 \times 10^{6}$.

Samples of Cz-Si were obtained from the same wafer with initial oxygen concentration $1.1 \times 10^{18} \mathrm{~cm}^{-3}$. All samples underwent a double "oxygen-nucleation" treatment, first at $450^{\circ} \mathrm{C}$ for $20 \mathrm{~h}$, then $20 \mathrm{~h}$ at $650^{\circ} \mathrm{C}$ and an additional treatment under high pressure at $870-950^{\circ} \mathrm{C}$. Details of the thermal treatments are given in Ref. [11].

Implanted samples were obtained from $p$-type (100) $\mathrm{Si}(1.7-2.5 \Omega \mathrm{cm})$, Czochralski-grown (about $6.6 \times 10^{17} \mathrm{~cm}^{-3}$ oxygen content) wafers, co-implanted at room temperature with $\mathrm{He}^{+}$ions at $30 \mathrm{keV}$ with a dose of $1 \times 10^{16} \mathrm{ions} / \mathrm{cm}^{2}$ and successively with $\mathrm{H}^{+}$ions at $24 \mathrm{keV}$ with a dose of $1 \times 10^{16}$ ions $/ \mathrm{cm}^{2}$. Details of the thermal treatment are given in Ref. [10].

\section{Results}

For molybdenum samples all three programs give similar $\tau_{1}$ values (see Table I), slightly overestimating the theoretical value for the defect-free metal (119 ps); the values from GA are closest to the theoretical value. On the other hand, POSFIT performs the best as far as the source background contribution is concerns - this package has no problems in finding correctly the $383 \pm 0.5$ ps lifetime for kapton with the exact $4 \%$ contribution. Both GA-MLR and LT programs tend to overestimate the $\tau_{2}$ lifetime (compensating it by a lower $I_{2}$ intensity). 
TABLE I

The comparison of the lifetimes calculated for molybdenum in the following programs: POSFIT (first row for every sample), LT (second row) and GA-MLR (third row) method. FWHM = $170 \mathrm{ps}$, the source contribution is estimated at $11 \%-382 \mathrm{ps}, \mathrm{GA}-$ MLR shift -6 channels.

\begin{tabular}{c|c|c|c}
\hline \hline Sample & $\tau_{1}[\mathrm{ps}]$ & $\tau_{2}[\mathrm{ps}]$ & $I_{2}[\%]$ \\
\hline M001 & 119.3 & 382.5 & 4.4 \\
& 119.8 & 408.7 & 3.6 \\
& 119.6 & 478.2 & 2.6 \\
M002 & 122.2 & 383.3 & 3.5 \\
& 122.7 & 433.1 & 2.8 \\
& 121.5 & 430.7 & 2.3 \\
M003 & 122.1 & 382.7 & 3.4 \\
& 123.1 & 457.0 & 2.4 \\
& 122.6 & 487.7 & 2.5 \\
M004 & 120.1 & 383.5 & 4.8 \\
& 120.3 & 394.5 & 3.7 \\
& 118.6 & 406.0 & 3.0
\end{tabular}

In Table II we show lifetimes for Cz-Si samples. LT and GA-MLR programs give similar results, in general not much different also from POSFIT analysis. All programs give values of $\tau_{1}$ ranging from about 226 to 231 ps. This is higher than the theoretical value of $220 \mathrm{ps}$ for non-defected Si [2]. This difference should, probably, be attributed to presence of oxygen-decorated defects. Both LT and GAMLR give similar values for $\tau_{2}$, somewhat higher than POSFIT. In compensation, POSFIT gives higher intensity $I_{2}$ of the second lifetime.

For $\mathrm{He}-\mathrm{H}$ implanted $\mathrm{Si}$, in spite of the poorer statistics, the two packages POSFIT and LT agree pretty well (see Table III). For near-to-surface implantation depth of positrons all three programs show shortening of the $\tau_{1}$ lifetime, below the value for pure Si. This is to be attributed to out-diffusing positrons towards sample surface. All three packages agree also well in determining the long lifetime component - both its value and intensity. GA-MLR fails only when the $I_{2}$ intensity becomes weak.

\section{Discussion}

Generally, the new GA-MLR method performs somewhat poorer than traditional packages (POSFIT and LT) for simple cases, like measurements with high 
TABLE II

The lifetimes calculated for the samples of Czochralski-grown silicon. POSFIT (first row for each sample), LT (second row) and GA- MLR (third row) $\mathrm{FWHM}=165 \mathrm{ps}$, the source contribution $11 \%$ - 382 ps, for GA-MLR "laser pulse" Gaussian shifted by -123 ps.

\begin{tabular}{c|c|c|c}
\hline \hline Sample & $\tau_{1}[\mathrm{ps}]$ & $\tau_{2}[\mathrm{ps}]$ & $I_{2}[\%]$ \\
\hline 71A0001 & 231.6 & 487.7 & 5.9 \\
& 227.4 & 1178.6 & 0.5 \\
& 228.3 & 1197.8 & 2.7 \\
W2E0001 & 222.8 & 425.7 & 13.6 \\
& 226.5 & 606.1 & 0.1 \\
& 221.2 & 1100.0 & 2.3 \\
W540005 & 230.3 & 459.9 & 8.6 \\
& 228.3 & 597.3 & 1.6 \\
& 227.8 & 798.2 & 1.7 \\
W77A0002 & 227.1 & 861.0 & 10.3 \\
& 228.6 & 858.2 & 1.3 \\
& 228.9 & 899.6 & 4.3
\end{tabular}

statistics in defect-free metals. As far the main lifetime component (i.e. the short one) is well identified by all three methods, the GA- MLR overestimates the source lifetime.

On the other hand, once fixed the source component, using the well known lifetime in kapton, GA-MLR performs even better than POSFIT, producing little scatter between different samples in Cz-Si. GA-MLR has again some problems with the long components, which is not a surprise taking into account not fully correct implementation of the resolution function in the present version of the program.

A weak point of the present application of GA-MLR is a simplified treatment of the resolution function. As the present version of the program is just a modification of the code applied for the fluorescence spectroscopy, the convolution of spectra with the resolution function is different in LT (and POSFIT) vs. GA-MLR. In GA-MLR program we mimic the resolution function by the "laser pulses". Obviously, this pulses approach works well just at the beginning of the spectra and does not correspond precisely to the convolution. Moreover, both the shift of the "laser" pulse as well its intensity must be carefully chosen.

From comparison of all three methods, a conclusion on the further need for development is clear. POSFIT performs very well but when the lifetimes show 
TABLE III

The lifetimes $\tau_{1}$ calculated for the samples of $\mathrm{He}+\mathrm{H}$ implanted Si. POSFIT (first row for every sample), LT (second row) $=230 \mathrm{ps,}$ GA-MLR (third row). Resolution function for POSFIT and LT 230 ps, for GA "laser pulse" shifted by -123 ps. "Sample" label gives the implantation energy of positrons.

\begin{tabular}{c|c|c|c}
\hline \hline Sample & $\tau_{1}[\mathrm{ps}]$ & $\tau_{2}[\mathrm{ps}]$ & $I_{2}[\%]$ \\
\hline $0.5 \mathrm{keV}$ & 140.7 & 436.3 & 90.4 \\
& 142.0 & 442.1 & 90.7 \\
& 145.1 & 447.2 & 85.8 \\
$2.5 \mathrm{keV}$ & 211.5 & 452.1 & 90.1 \\
& 212.0 & 456.7 & 92.0 \\
& 210.0 & 450.1 & 93.5 \\
$5 \mathrm{keV}$ & 223.3 & 488.5 & 63.5 \\
& 223.6 & 477.0 & 69.9 \\
& 222.7 & 481.7 & 38.5 \\
& 223.5 & 492.6 & 17.8 \\
& 224.5 & 484.1 & 22.1 \\
& 227.1 & 471.9 & 54.2
\end{tabular}

pretty distinguishable values and strong intensities. The LT program shows a wide range of the convergence, i.e. allows working with unknown starting parameters. GA-MLR is particularly efficient in guessing short lifetimes. Future developments should combine these strong features of all three methods.

\section{Ackowledgments}

Two of us (A.K. and G.K.) acknowledge support from Rector's UMK grants: No. $354 \mathrm{~F} / 2007$ and $778 \mathrm{~F} / 2006$.

\section{References}

[1] G.P. Karwasz, A. Zecca, R.S. Brusa, D. Pliszka, J. Alloys Comp. 382, 244 (2004).

[2] M. Hakala, M.J. Puska, R.M. Nieminen, Phys. Rev. B 57, 7621 (1998).

[3] I. Itoh, H. Murakami, A. Kinshita, Appl. Phys. Lett. 63, 2798 (1993).

[4] S. Yang, Y. Li, Q. Ma, H. Liu, Q. Hao, Y. Li, S. Niu, H. Li, J. Cryst. Growth 276, 43 (2005).

[5] P. Banerji, Appl. Surf. Sci. 253, 5129 (2007). 
[6] J.J. Fisz, J. Phys. Chem. A 110, 12977 (2006).

[7] U. Rodemerck, M. Baerns, M. Holena, Appl. Surf. Sci. 223, 168 (2004).

[8] J.N. Cawse, M. Naerns, M. Holena, J. Chem. Inf. Comput. Sci. 44, 143 (2004).

[9] K. Gozdziewski , A. J. Maciejewski, C. Migaszewski, Astrophys. J. 657, 546 (2007).

[10] R.S. Brusa, W. Deng, G.P. Karwasz, A. Zecca, D. Pliszka, Appl. Phys. Lett. 79, 1492 (2001).

[11] G.P. Karwasz, R.S. Brusa, A. Misiuk, A. Zecca, Acta Phys. Pol. A 95, 575 (1999).

[12] P. Kirkegaard, M. Eldrup, Comput. Phys. Commun. 3, 240 (1972).

[13] R.S. Brusa, C. Macchi, S. Mariazzi, G.P. Karwasz, W. Egger, P. Sperr, G. Kgel, Appl. Phys. Lett. 88, 011920 (2006).

[14] J. Kansy, Nucl. Instrum. Methods Phys. Res. A 374, 235 (1996).

[15] J. Kansy, Mater. Sci. Forum 652, 363 (2001). 